\title{
Essai d'interprétation fonctionnelle des entités spatiales structurées du village de Courtisigny (XIIIe-XVe siècles). Pistes sur une réflexion en \\ cours
}

Attempt of functional interpretation of the structured spatial entities of the village of Courtisigny (14th-15th centuries). Survey of a work in progress Intento de interpretación de la prueba funcional de las entidades espaciales estructuradas de la aldea de Courtisigny (siglos XIII-XV) Pistas para una reflexión en curso

Ein Versuch einer funktionalen Interpretation strukturierter räumlicher Siedlungseinheiten des Dorfes von Courtisigny (14.-15. Jh. n. Ch.). Ein Überblick über die laufenden Untersuchungen

\section{Claire Hanusse et Stéphanie Dervin}

\section{OpenEdition \\ Journals}

Édition électronique

URL : https://journals.openedition.org/rao/4538

DOI : $10.4000 /$ rao. 4538

ISBN : 978-2-7535-5014-8

ISSN : 1775-3732

Éditeur

Presses universitaires de Rennes

\section{Édition imprimée}

Date de publication : 31 décembre 2015

Pagination : 385-399

ISBN : 978-2-7535-5012-4

ISSN : 0767-709X

Référence électronique

Claire Hanusse et Stéphanie Dervin, « Essai d'interprétation fonctionnelle des entités spatiales structurées du village de Courtisigny (XIIIe-XVe siècles). Pistes sur une réflexion en cours ", Revue archéologique de l'Ouest [En ligne], 32 | 2015, mis en ligne le 28 avril 2018, consulté le 23 août 2022. URL : http://journals.openedition.org/rao/4538; DOI : https://doi.org/10.4000/rao.4538

Ce document a été généré automatiquement le 23 août 2022.

Tous droits réservés 


\title{
Essai d'interprétation fonctionnelle des entités spatiales structurées du village de Courtisigny (XIIIe- XVe siècles). Pistes sur une réflexion en cours
}

\author{
Attempt of functional interpretation of the structured spatial entities of the \\ village of Courtisigny (14th-15th centuries). Survey of a work in progress \\ Intento de interpretación de la prueba funcional de las entidades espaciales \\ estructuradas de la aldea de Courtisigny (siglos XIII-XV) Pistas para una \\ reflexión en curso \\ Ein Versuch einer funktionalen Interpretation strukturierter räumlicher \\ Siedlungseinheiten des Dorfes von Courtisigny (14.-15. Jh. n. Ch.). Ein Überblick \\ über die laufenden Untersuchungen
}

Claire Hanusse et Stéphanie Dervin

1 «L'interprétation des mobiliers mis au jour en contexte d'habitat représente toujours un défi, dès lors que nous choisissons d'atteindre les habitants de nos villages " affirmait Jean-Marie Pesez. Que nous tentions de donner une dimension économique ou sociale à nos découvertes, il nous faut préciser les indicateurs sur lesquels se fondent nos hypothèses et nos propositions, pour maitriser mieux les limites et les potentiels réels de notre documentation sur ces thèmes. S. Smith nous rappelait en effet récemment que : "It is important, however, that archaeologists do not abandon the study of these matters [les thématiques économiques et sociales] to historians; we need to recognize that both documents and material culture played a part in producing and reflecting the realities of peasant existence, and that both were involved in power relationships and in the creation of social life. These evidence types need to sit side-by-side one another, with one having no more explanatory force than the other ${ }^{1}$.» (Smith 2009 p. 393-394). C'est une évidence pour les 
archéologues travaillant sur les périodes historiques, mais nous sommes également tentées d'ajouter : sans que le cadre d'explication nous soit imposé par une lecture au premier degré, pour ne pas dire primaire de nos données, comme cela nous fut suggéré il n'y a pas si longtemps (Bougard, Noyé, 2003).

2 La présence de ces mobiliers (céramique, objets métalliques, etc.), résulte d'un processus complexe - mis en relation avec le cadre de vie - qui commence avec l'usage de ces objets, leur réutilisation plus ou moins systématique, leur recyclage éventuel qui leur donne une seconde vie en changeant éventuellement leur fonction initiale, leur rejet et enfin les conditions de leur conservation post-dépositionnelle. C'est un rappel de principe qui situe les limites de la documentation avec lesquelles nous composons.

3 À partir de l'exemple des fouilles du village déserté de Courtisigny, nous souhaitons caractériser en tant qu'unités fonctionnelles ce que nous avons dénommé des « Entités Spatiales Structurées" (ESS), une terminologie volontairement à faible valeur interprétative. Après avoir situé le contexte archéologique nous allons tenter de répondre à deux questions : 1) Comment peut-on caractériser ces ESS ? 2) Comment interpréter les différences constatées?

\section{Le village de Courtisigny}

4 Le site du village de Courtisigny, localisé au lieu-dit «Fosses Saint-Ursin » sur la commune de Courseulles-sur-mer, est implanté à 3,5 kilomètres des côtes de la Manche et à une quinzaine de kilomètres au nord-ouest de l'agglomération caennaise, dans un paysage ouvert probablement très proche de celui des $\mathrm{XIV}^{\mathrm{e}}-\mathrm{XV}^{\mathrm{e}}$ siècles. L'analyse de la documentation archéologique mise au jour lors des fouilles permet de démontrer que l'on a affaire à un lieu occupé anciennement, depuis le haut Moyen Âge au moins, voire dès le Bas-Empire. Attesté sous le nom de Courtisigny dans la documentation textuelle, au milieu du $\mathrm{XII}^{\mathrm{e}}$ siècle, ce village appartient à la seigneurie de Courseulles qualifiée, comme c'est devenu l'usage à partir du xive siècle, de baronnie de CourseullesBernières. La plus ancienne mention d'un seigneur de Courseulles est celle de Roger (Rogerio de Cursella), qui apparaît dans une donation effectuée au Xi siècle, sous l'égide du duc Guillaume, en faveur de la cathédrale de Bayeux. La présence d'un édifice de culte remplissant des fonctions paroissiales, et le caractère aggloméré de cet habitat permettent sans discussion de qualifier cet ensemble de village. Un modeste village, certes, dont les fonctions économiques sont principalement sinon exclusivement agricoles, car il n'a accueilli aucune activité artisanale spécialisée, en l'état actuel de la documentation; aucune fonction administrative ne lui est attribuée dans la documentation textuelle, si ce n'est bien sûr d'être jusqu'au XIv ${ }^{e}$ siècle au moins le centre d'une paroisse située au diocèse de Bayeux.

5 Les habitants de ce village, qui tirent leurs ressources du travail de la terre, sont donc principalement des paysans - c'est un truisme - mais constituent néanmoins une catégorie hétérogène par la diversité de leurs statuts, leurs capacités économiques : du manouvrier se plaçant au jour le jour, du valet au service d'un paysan plus ou moins fortuné qui vit sous le même toit et avec la famille de son "patron ", au descendant du vavasseur attesté au XIII ${ }^{\mathrm{e}}$ siècle, incarnant une modeste élite rurale dont certains se transforment peu à peu «en coqs de village », ces hommes et leurs familles vivant au village de Courtisigny, constituent un petit monde «a small world», pour reprendre le 
titre d'un fameux ouvrage de Wendy Davies sur la région de Redon (Davies 1988). Autrement dit, une communauté multiforme ancienne qui se reconnaît dans ses pratiques religieuses, ses pratiques agraires, les expériences partagées, ici comme ailleurs dans un rapport de dépendance que l'on ne saurait cependant réduire à la seule relation au seigneur. Dans ce paysage ouvert, dominé par la céréaliculture associée notamment à l'élevage du mouton, l'économie de ce village se situe, à la fin du Moyen Âge, dans un contexte marqué par le renversement de la tendance qui fait entrer l'Occident dans une conjoncture de crise systémique. Celle-ci a contribué à une réorganisation de l'espace agraire et à l'effacement progressif de lieux habités tels que celui de Courtisigny, au terme d'un processus de plusieurs décennies, voire de quelques siècles.

\section{La documentation}

$6 \quad$ Les données sur lesquelles portent nos analyses sont issues d'un site fouillé en deux temps, au début des années soixante-dix à l'occasion de travaux académiques (chantiers 1 à 3), puis, de 1999 à 2008, lors d'une fouille programmée pluriannuelle (zones 1 à 7 ; fig. 1). D'abord inscrites dans le cadre de la problématique des habitats désertés qui caractérisaient les enquêtes situées dans le prolongement des travaux emblématiques de Wharram Percy, Dracy ou Rougiers, les fouilles se sont ensuite insérées dans une perspective renouvelée, prenant en compte les acquis de l'archéologie préventive (Hanusse, 2012). Les quelques deux mille mètres carrés fouillés, qui ne représentent qu'une partie du site qui pourrait occuper jusqu'à trois hectares dans sa plus large extension, ont permis de mettre au jour les vestiges d'un cimetière associé à ce que nous interprétons comme les fondations de l'église, et surtout, à une cinquantaine de mètres plus au nord, sur le versant d'une petite vallée sèche dont le profil au bas Moyen Âge, résulte largement de l'extraction des matériaux de construction ${ }^{2}$, les vestiges de bâtiments structurés autour d'un chemin est-ouest. De part et d'autre de cet axe, se développent des unités habitées composées de cours et de bâtiments, sur les caractéristiques desquelles nous allons revenir. Au nord du chemin, un espace ouvert, une cour insérée dans le bâti, accueille l'unique puits découvert à ce jour sur le site. Ces bâtiments construits de moellons calcaire tirés de la plaquette extraite in situ, comme cela est fréquemment le cas dans les habitats ruraux de la région postérieurs au $\mathrm{XII}^{\mathrm{e}}$ siècle, étaient couverts de matériaux périssables, du chaume dénommée «glieu » dans la documentation textuelle (Hanusse et Jarry, 2007). Seule l'église semble avoir reçu une couverture d'ardoises lors de la phase ultime de son fonctionnement, comme en témoignent les accumulations de ces matériaux à son emplacement présumé. 
Figure 1 : Plan d'ensemble du site des « Fosses Saint-Ursin », à Courseulles-sur-Mer (Calvados) Figure 1: Map of the "Fosses Saint-Ursin" settlement, Courseulles-sur-Mer (Calvados).
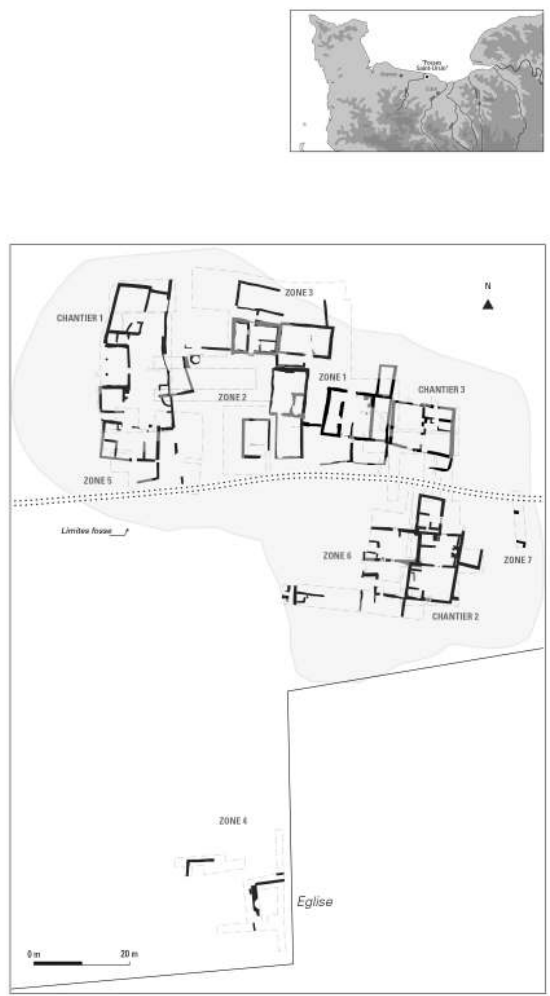

7 Nous avons caractérisé des ensembles que nous avons qualifiés d'« entités spatiales structurées" (ESS) équivalentes à des unités parcellaires (fig. 2). La pratique de l'extraction, dans les limites des terrains concédés, est très aisément repérable et détermine des paliers/terrasses suivant la pente du versant qui contribuent à un premier repérage d'unités spatiales. La localisation des portes des bâtiments, l'identification de zones de circulation participant aux liaisons entre ces différents espaces et structures, contribuent ensuite à l'individualisation et à l'interprétation des ESS de dimensions et de compositions très variables : certaines comprennent un seul bâtiment situé entre deux cours, d'autres plusieurs répartis autour des cours selon des dispositifs variés. 


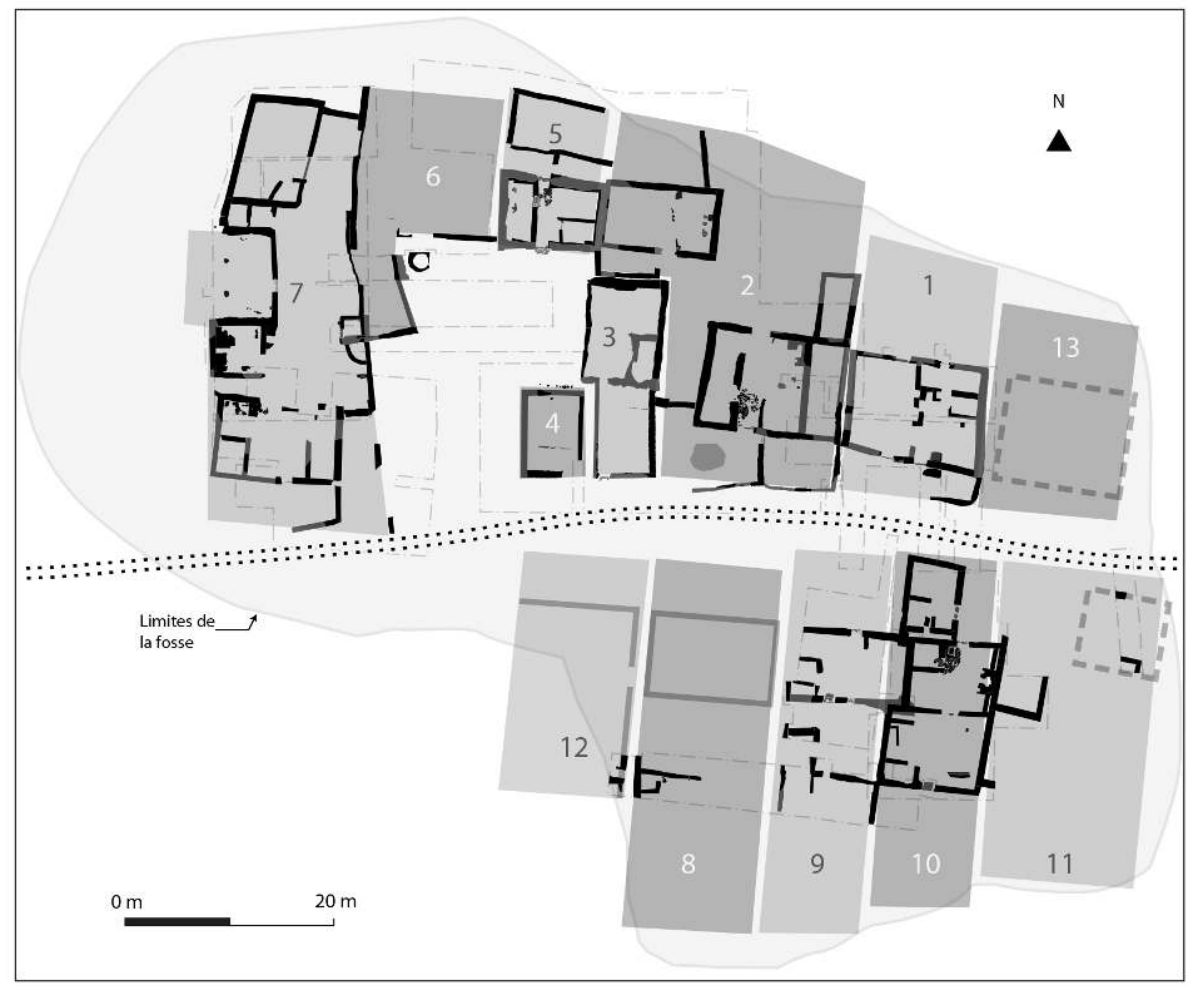

$\mathrm{Au}$ total nous avons pu repérer à partir des indices archéologiques et/ou topographiques quatorze ESS fouillées intégralement, partiellement ou simplement localisées, dont treize dans la partie nord du site. Le site en comptait d'autres qui ont été enfouies dans la fosse après les premières fouilles par le propriétaire des lieux elles sont lisibles sur des photographies aériennes faites au moment de ces premiers travaux - et nous supposons la présence d'autres unités au sud-est de la fosse au regard des matériaux remontés par les labours dans les parcelles limitrophes qui ont été régulièrement prospectées. Sur le chemin, ces ESS occupent entre dix et dix-sept mètres de large, sur une longueur qui varie de quinze mètres à plus de quarante mètres. Dans le meilleur des cas, nous sommes capables de restituer des états successifs de ces ESS ou d'une partie de celles-ci qui peuvent occuper de moins de cinquante mètres carrés à plus de six cents mètres carrés.

9 L'analyse de ces entités, leur composition, leur organisation, contribuent à mieux cerner le fonctionnement du site, bien que ces recompositions ne soient décelables que grâce à une lecture stratigraphique horizontale. La nature des matériaux utilisés et surtout le mode d'implantation du bâti aménagé directement sur le substrat calcaire entaillé lors de la construction, en sont la cause. La sédimentation des occupations est donc faible dans les bâtiments parfois dotés de sols de terre battue soigneusement entretenus, comme hors des bâtiments, dans les cours dont les sols recouvrent de quelques centimètres à peine le substrat calcaire après extraction ${ }^{3}$.

Deux catégories de bâtiments se distinguent : des maisons d'habitation principalement caractérisées par la présence d'au moins un foyer associé à des niveaux interprétables comme des sols d'usage, et des bâtiments d'exploitation caractérisés le plus souvent en négatif des maisons d'habitation, et plus rarement de manière positive par la présence 
d'aménagements (fosse de métier à tisser, propreté des sols, dimensions et disposition des bâtiments, etc.) témoignant d'autres activités dont la juxtaposition, complétée par une analyse du mobilier céramique et métallique qui leur est associé, contribue ou pas à l'identification d'unités fonctionnelles. C'est tout au moins ce que nous souhaitons évaluer dans le cadre de cet article, en confrontant les données issues de deux des ESS que nous avons choisies pour la qualité de l'information qu'elles regroupent. Ces ESS se situent au nord du chemin structurant (fig. 2) : la première, ESS 7, occupe au moins six cents mètres carrés; la seconde, l'ESS 5, environ cent cinquante mètres carrés, est localisée au fond d'une vaste cour largement ouverte sur le chemin au sein de laquelle se trouve le puits interprété comme communautaire, qui suppose que cette cour n'appartient pas à l'ESS 5.

11 Les états sur lesquels nous pouvons travailler résultent d'agrandissements et de restructurations qui ont pu éventuellement transgresser des limites de parcelles anciennes, dont rend compte l'analyse détaillée de chacun de ces ensembles (Hanusse, 2012). Le plan d'ensemble que nous donnons (fig. 2) est un document de synthèse qui rassemble les treize ESS de la fosse dans leur état final, dont l'ultime phase de fonctionnement n'est pas strictement contemporaine, car celle-ci s'étend au long des $\mathrm{XIV}^{\mathrm{e}}$ et $\mathrm{XV}^{\mathrm{e}}$ siècles. Le mobilier associé est considéré comme représentatif, même si nous gardons à l'esprit que cette documentation se compose d'assemblages dont la constitution s'inscrit dans un temps plus long que l'instantané relatif à partir duquel nous raisonnons ${ }^{4}$.

\section{Le bâti comme indicateur}

12 La composition et l'organisation des ESS 5 et 7 est très différente (fig. 3 ) : la plus grande (ESS 7) associe à un grand bâtiment de 95 mètres carrés hors tout (bat 12), une grande unité habitée, et un vaste ensemble aménagé perpendiculairement au chemin principal comprenant, outre la maison donnant sur une cour bordant le chemin, une vaste cour fermée à l'arrière, le long de laquelle se développe à l'ouest une file de quatre bâtiments de dimensions inégales. Un sondage réalisé durant des travaux pratiques au cours des années soixante-dix, indique la relation de cette structure avec un autre bâtiment mitoyen situé à l'ouest, mais qui n'a pas été fouillé, suggérant une extension de cette ESS. Un bâtiment (bat 17), le plus proche de la maison principale (bat 12), contient les vestiges d'un petit foyer ouvert associés à un sol de terre battue souillé de quelques déchets : il s'agit à n'en pas douter d'une petite unité d'habitation de moins de seize mètres carrés. Le bâtiment $15\left(78 \mathrm{~m}^{2}\right)$ qui occupe l'angle nord-ouest de la cour, et ouvre sur celle-ci, est interprétable, compte tenu de la rareté du mobilier, de la propreté du sol, comme un bâtiment d'exploitation à la fonction imprécise : grange, remise que l'aménagement d'un plan incliné rendait plus accessible. Le bâtiment $16\left(67 \mathrm{~m}^{2}\right)$ n'a pas été fouillé intégralement, l'emplacement de son extrémité occidentale reste sujet à discussion puisqu'il se prolonge sous la banquette ouest. Il ouvre sur la cour 6 par un seuil barré, un dispositif commun sur le site. La présence d'un métier à tisser dans l'angle nord-est (une interprétation des premiers fouilleurs) est plausible. Le sol propre de ce bâtiment incomplètement fouillé n'indique pas un usage domestique, mais plutôt un espace de travail et de stockage : une grange vraisemblablement, ce que conforte la structuration de l'espace en trois nefs, fréquente dans ce type d'installation. L'analyse des données suggère une ouverture de cette grange plus à l'ouest, au-delà de l'espace fouillé, car le passage situé au sud du bâtiment 17 ne permettait pas la circulation de 
charrettes. Dans cette cour, adossées au mur de clôture est, deux petites structures de faible superficie $\left(<2 \mathrm{~m}^{2}\right)$, ont été interprétés notamment comme des niches. Contre le bâtiment 12 ou sous le même toit, une pièce (PCE 2), dont le mur occidental est mitoyen avec un mur sur lequel est aménagée une vaste cheminée (1,50 $\mathrm{m}$ d'ouverture), au centre de la pièce principale, a été hypothétiquement interprétée comme une écurie, du fait notamment de la propreté du sol.

Figure 3 : Plan des ESS 5 et 7 Figure 3: Map of ESS5 et ESS5.
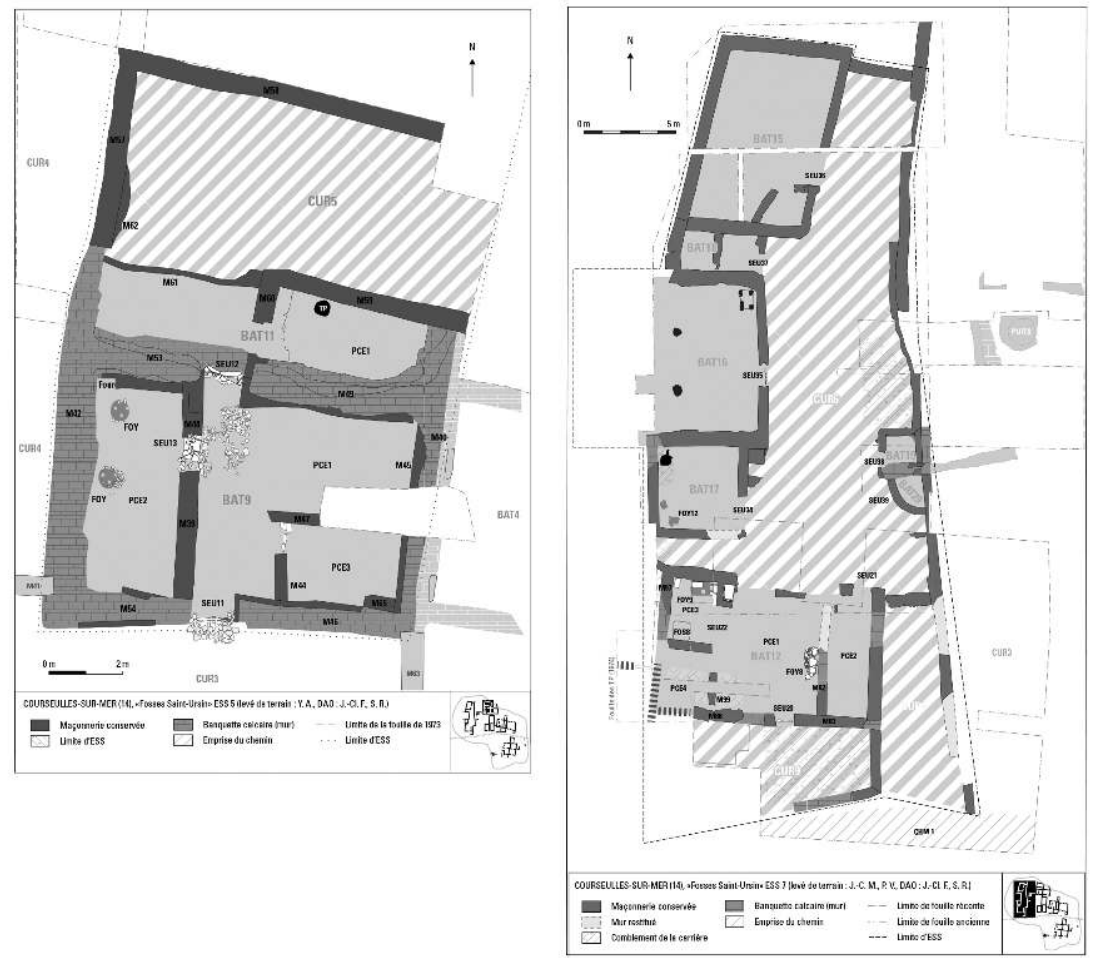

L'ESS 5 se présente d'une tout autre manière, puisqu'elle ne comprend qu'un bâtiment plus modeste $\left(<50 \mathrm{~m}^{2}\right)$, structuré selon des principes bien attestés sur le site, autour d'un couloir traversant sur lequel donnent trois pièces. L'une, la plus grande, contient deux foyers ayant fonctionné successivement et un petit four (potager ?) aménagé dans le mur nord, formant de fait une cheminée qui assure l'aération de cette pièce de petite taille. Le couloir donne à l'arrière sur une cour close et à l'avant sur une vaste cour «publique ». Un petit appentis ( $13 \mathrm{~m}^{2}$ hors tout mais seulement $5 \mathrm{~m}^{2}$ utiles) est adossé au nord, au bâtiment principal interprété hypothétiquement comme une écurie.

La distinction fonctionnelle des bâtiments se fonde sur la nature des niveaux d'occupation, la présence de foyers ouverts plus ou moins importants, des dispositifs que l'on considère comme des éléments de confort tels des placards aménagés dans les murs, ou encore des fours, des fosses de stockage, d'un aménagement interprété comme l'emplacement de métier à tisser (ESS 7), ce qui indique un usage différencié des espaces. Ceci nous a encouragés à distinguer maisons d'habitation et bâtiments d'exploitation. La rapide description de ces deux ensembles suggère sur la base d'une simple comparaison de leurs compositions respectives, un premier niveau de différentiation. 


\section{La céramique comme indicateur}

15 L'étude céramologique réalisée sur l'ensemble du site repose sur un système d'enregistrement permettant le comptage par unité stratigraphique et par structure. Les découvertes réalisées au cours des années soixante-dix (chantier 1 qui recoupe une partie de l'ESS 7) ont en revanche été enregistrées selon un carroyage distinguant les différents contextes. Malgré ces disparités, l'enregistrement et la quantification sont suffisamment cohérents pour permettre l'analyse de ces ensembles dans un même système typo-chronologique actualisé, fondé sur les différents groupes techniques et la morphologie dont les principes ont été présentés par ailleurs (Dervin, Hanusse et Bocquet-Liénard, 2013). L'étude du mobilier céramique a donc permis de proposer une typo-chronologie qui a contribué au phasage du site, en particulier pour le bas Moyen Âge, phases $2 \mathrm{~B}$ à $2 \mathrm{E}$ qui couvrent une fourchette chronologique allant de la fin $\mathrm{XI}^{\mathrm{e}}$ début $\mathrm{XIII}^{\mathrm{e}}$ siècle jusqu'au $\mathrm{XV}^{\mathrm{e}}$ siècle. La répartition du mobilier céramique appartenant à ces phases (soit environ $72 \mathrm{~kg}$ ) n'est pas homogène sur l'ensemble de notre espace d'étude puisque d'une structure à l'autre (bâtiments ou cours), le poids peut varier de quelques grammes (quelques tessons) à plus de treize kilos (fig. 4). Ces contrastes résultent d'abord des processus de sédimentation et surtout du contexte de découverte, dont nous avons évoqué la diversité en introduction. La répartition de ce mobilier céramique n'est donc pas uniforme et ne dépend pas de la superficie occupée par les unités à l'intérieur desquelles s'établit le décompte. Le constat est analogue à l'échelle des bâtiments et des cours, comme à celle de nos ESS. En effet, la masse des céramiques ne dépend pas de la surface au sol des ESS identifiées : dans l'ESS 7 nous comptabilisons près de neuf kilos de tessons alors que l'ESS 5 , qui occupe une surface quatre fois moins grande, regroupe plus de dix kilos, principalement mis au jour dans un dépotoir situé au fond de la cour 5 . 
Figure 4 : Répartition par poids des céramiques mises au jour sur le site Figure 4: Distribution by weight of the pottery discovered on the site.

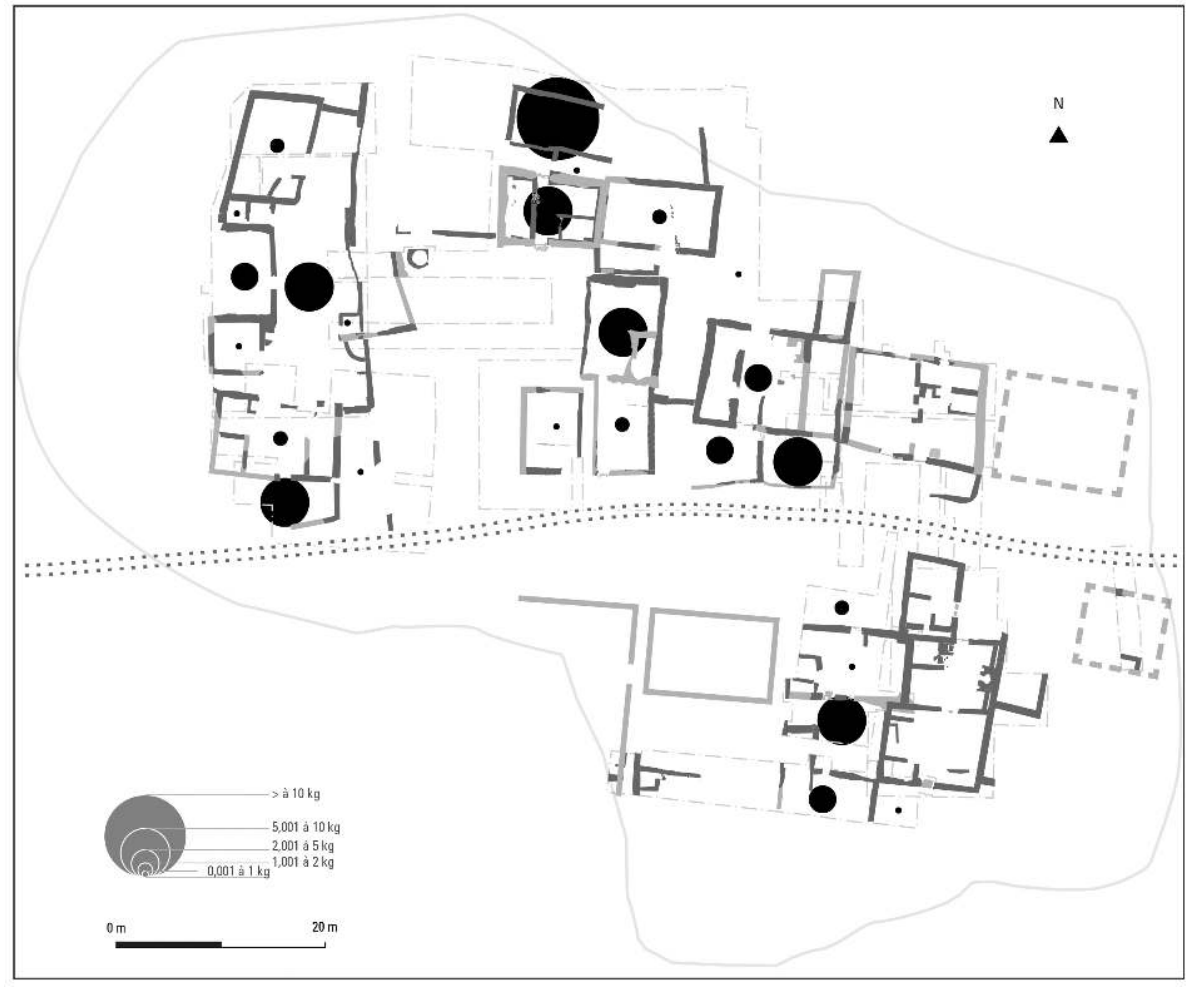

Au sein de ces deux ESS le mobilier céramique provient à $50 \%$ et plus des cours dont la taille et la morphologie sont fort différentes; certaines sont ouvertes vers l'extérieur de l'ESS 7, tandis qu'une est seulement reliée à la maison d'habitation (ESS 5). Dans les bâtiments, la céramique a été principalement mise au jour dans les pièces comprenant des foyers. Localisation logique car la présence de ce matériel résulte de l'intensité de l'activité quotidienne au plus près d'un équipement essentiel au cœur de la maison.

Si nous abordons maintenant ce mobilier d'un point de vue qualitatif, le constat s'impose d'une grande similitude à la fois des formes et de la typologie des poteries issues de ces deux ESS, notamment pour la céramique qui appartient à la dernière phase d'occupation (2E), la mieux représentée quantitativement (fig. 5). Il s'agit, à hauteur de $75 \%$ à $90 \%$, de matériel provenant de la zone de production du Bessin, située à 15-20 kilomètres à l'ouest du site. Cette production se caractérise par des pâtes claires fines glaçurées que l'on observe sur des formes fermées telles que des pots, des pots à dispositif rapporté (Flambard Héricher, 2008), des pichets/cruches, mais aussi des formes ouvertes de type jatte et poêlon. Quelques pots à pâte rouge se rattachent à la même aire de production, ainsi que des grès qui prennent la forme de pichets/ cruches. La grande majorité des céramiques retrouvées est donc d'origine locale (Dervin, Hanusse et Boquet-Liénard, 2013). Toutefois, la phase ultime d'occupation du site, qu'illustrent ces deux ESS, voit émerger sur ce site des productions du Pays d'Auge, situé plus à l'est au-delà de l'Orne. Leur apparition au sein de ces contextes marque aussi un changement dans l'approvisionnement en céramique, que l'on retrouve sur d'autres sites de la plaine de Caen et en particulier sur les sites d'utilisation caennais des $\mathrm{XIV}^{\mathrm{e}}$ et $\mathrm{XV}^{\mathrm{e}}$ siècles, comme celui de Montoir-Poissonnerie (Bocquet-Liénard et al., 2011). Le matériel de Courtisigny dans son ensemble, et de ces deux ESS en particulier, témoignent donc d'usages de consommation inscrits dans un réseau 
d'approvisionnement local, commun à d'autres sites de cet espace géographique de la plaine de Caen. Néanmoins, une observation plus fine des différentes catégories de vases présentes dans ces ESS, permet de noter, par comparaison avec la céramique mise au jour en contexte urbain, à Caen, l'absence ou la moindre proportion de certaines catégories de poteries. C'est le cas notamment des grands pots de stockage en grès du Mortainais/Domfrontais ou du Bessin, que l'on assimile à des pots à beurre. Si l'absence de ces pots peut s'expliquer par la distance séparant Courtisigny du lieu de production ${ }^{5}$, un tel argument ne saurait être sollicité sur notre site pour justifier le manque de grands vases fabriqués dans le Bessin.

Figure 5 : Les céramiques représentatives des ESS 5 et 7 Figure 5: Typical pottery of the ESS 5 and 7.

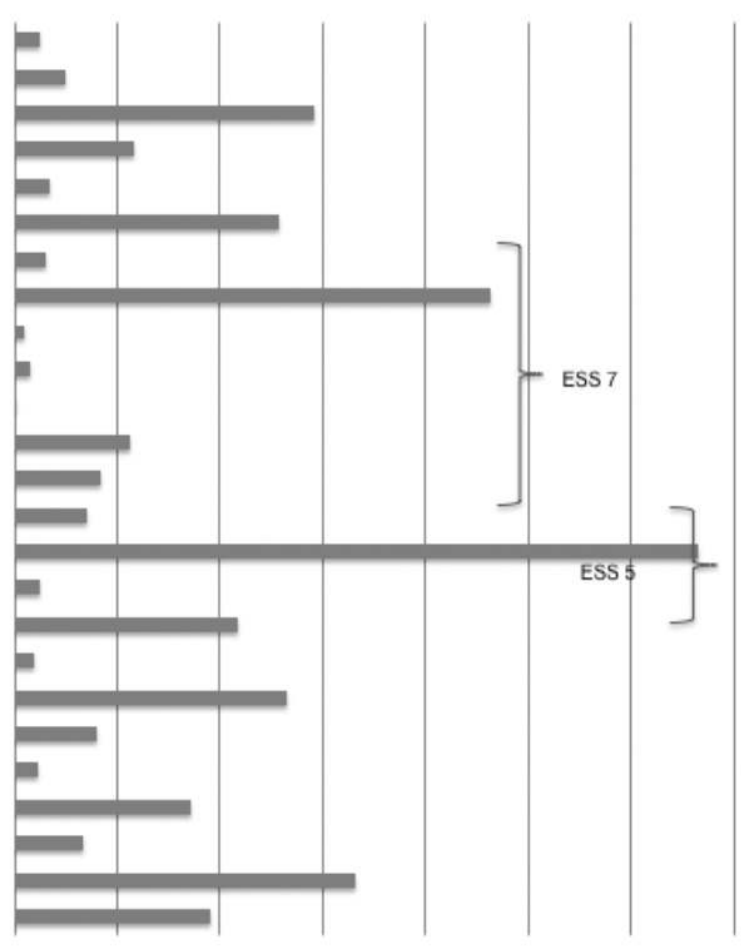

Si nous nous penchons maintenant sur la répartition des différentes catégories morphologiques dans chacune des structures composant les ESS, nous pouvons tenter de caractériser l'usage de ces structures, en nous fondant sur les assemblages de vases (présence/absence/proportion). Dans l'ESS 5, le bâtiment 9 et la cour 5 présentent un assemblage très proche (fig. 6), indiquant ainsi que la cour 5 est le seul espace de rejet utilisé par le bâtiment 9. Dans l'ESS 7, l'assemblage morphologique du bâtiment 12 apparaît similaire à celui du bâtiment 9 , ce qui témoigne d'un fonctionnement partiellement synchrone de ces espaces. De plus, le lien entre les bâtiments et les cours, concrétisé par les recollages de tessons retrouvés dans les bâtiments, notamment à proximité des foyers, avec d'autres découverts dans les cours (avant ou arrière), démontrent que ces dernières constituent des lieux de dépotoirs privilégiés, au moins initialement, notamment dans l'ESS 5, dotée d'une seule cour fermée à l'arrière de la maison d'habitation. Si des recollages existent entre le mobilier provenant du bâtiment 12 et les cours 6 et 9 qui l'enserrent au nord et au sud, les assemblages de ces deux espaces diffèrent cependant : pots, pots à dispositif rapporté et 
pichet/cruche sont plus nombreux dans la cour 6 , alors que les jattes sont plus abondantes dans la cour 9 .

Figure 6 : Proportions des différentes formes céramiques présentes dans l'ESS 5 Figure 6: Proportions of the various vessel forms in the ESS 5.

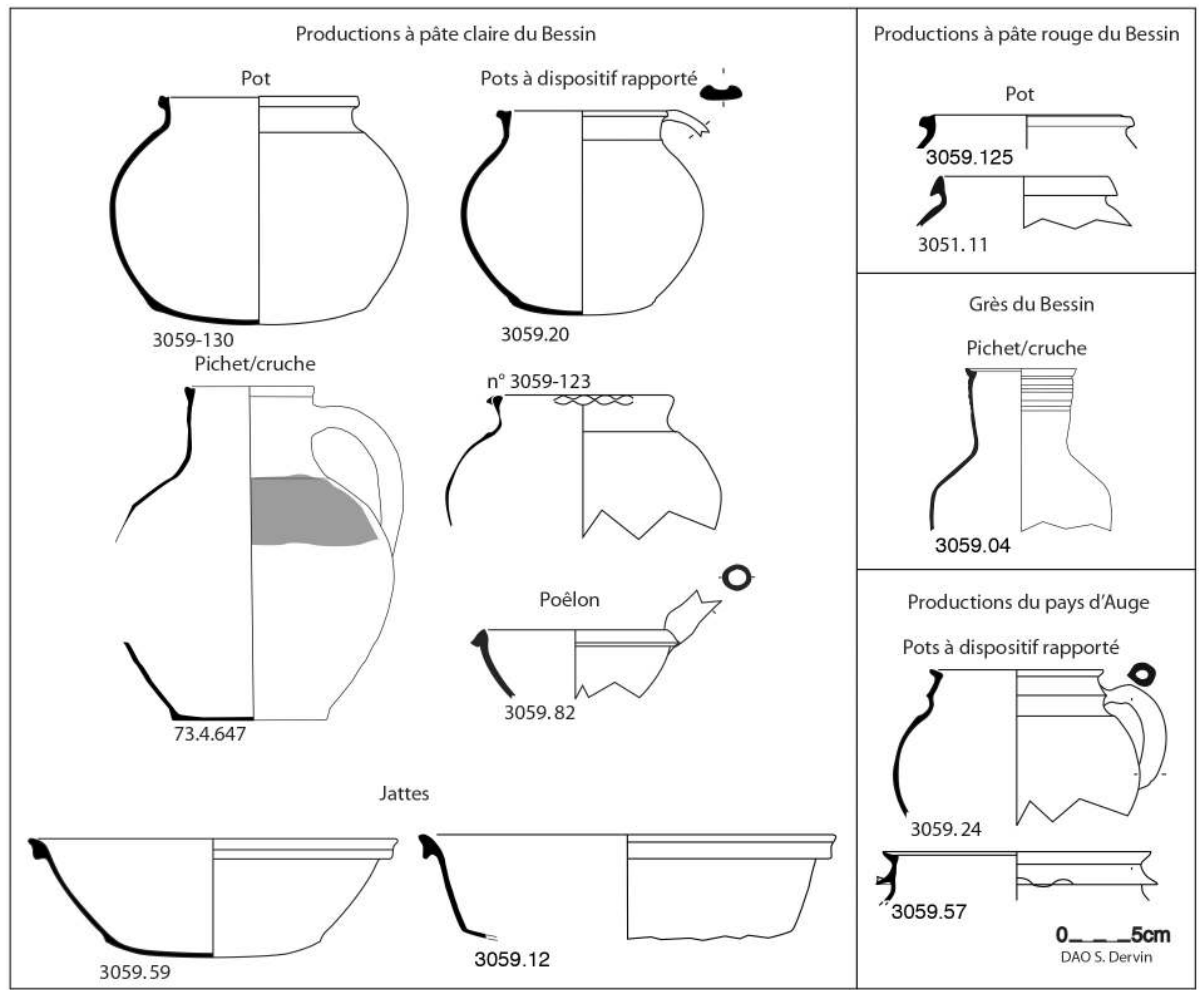

19 Le premier assemblage semble dominé par des spécimens morphologiquement interprétables comme des vases destinés à la cuisson ou au stockage des aliments et liquides, tandis que le second rassemble plutôt des formes liées à la préparation des aliments. (fig. 7). Comment expliquer ces différences? 
Figure 7 : Proportions des différentes formes céramiques présentes dans l'ESS 7 Figure 7: Proportions of the various vessel forms in the ESS 7.

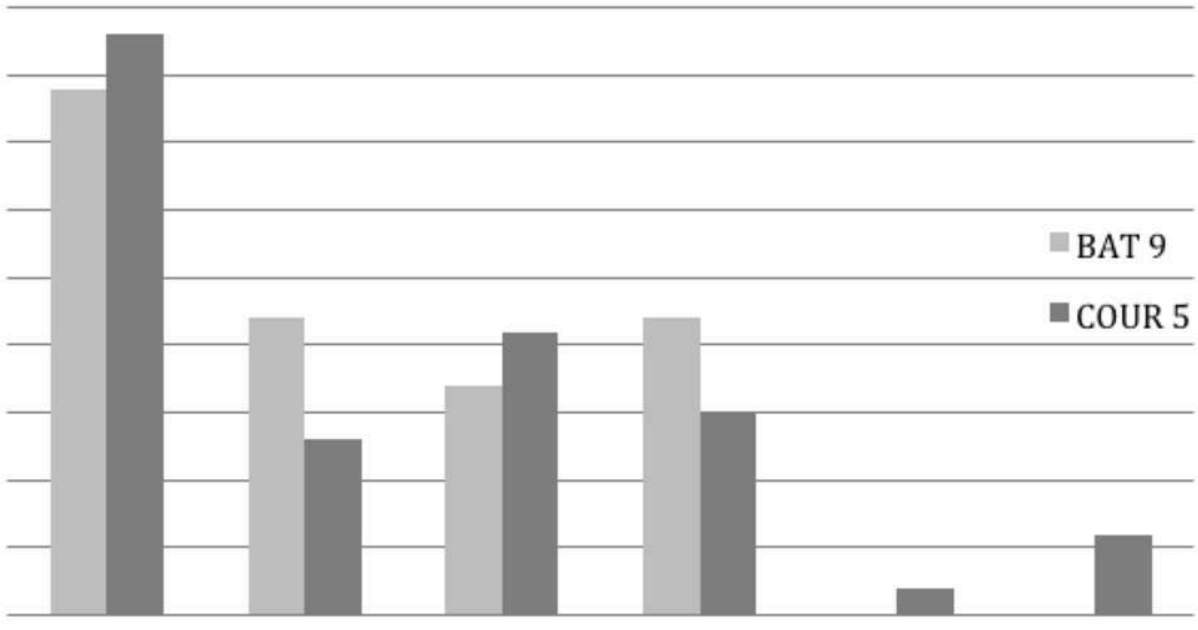
distinctes entre les deux cours pourraient être mises en rapport avec la fonction des pièces ouvrant sur celle-ci, explication tentante, mais que l'on peine à étayer. Cette maison possède une cuisine située au nord; la pièce principale, dotée d'une vaste cheminée murale, ouvre tout autant sur les deux cours. Sur la cour 6, donnent d'autres bâtiments dans lesquels la céramique est rare, à l'exception du bâtiment 15 qui renfermait quelques exemplaires (deux pots à dispositif rapporté et un pichet/cruche) ; il est interprété comme un bâtiment d'exploitation. Le bâtiment 17, en revanche, est équipé d'un foyer ouvert et, de fait, peut être interprété comme un modeste deuxième bâtiment d'habitation, mais on pourrait également à songer une seconde cuisine (?). Il reste difficile de dépasser ce cadre explicatif. Cependant, malgré ces limites, l'analyse conforte le constat d'une grande proximité entre les espaces d'utilisation de la céramique et ceux dédiés aux rejets, que l'on peut donc considérer comme représentatifs de la céramique consommée lors des phases d'occupation identifiées.

$21 \mathrm{Au}$ terme de cette rapide présentation du mobilier céramique, il ressort que ces artefacts, s'ils contribuent bien à caractériser l'occupation d'une partie des espaces habités, ne permettent pas, en revanche, de dessiner un tableau discriminant de la consommation de la céramique au sein de ces deux ESS.

\section{Le mobilier métallique comme indicateur}

Le mobilier métallique se prête par nature à un décompte en NMI simple, quelles que soient les difficultés d'identification que l'on rencontre et la diversité des contenus que cette notion recouvre. Compte tenu de l'ampleur limité de notre corpus, nous utiliserons cette variable dans son acception la plus simple (Demierre et al., 2013). Le pourcentage de mobilier indéterminé est souvent très élevé, entre 25 et $30 \%$ en NR, alors que de nombreux objets, ambivalents, restent non identifiés : des tiges cassées, des plaques et des fragments divers, informes, témoignent par leur présence de 
l'importance des mobiliers métalliques, en fer ou en alliage cuivreux, dans les habitats de cette époque, mais sont peu utiles lorsqu'il s'agit d'éclairer les activités quotidiennes.

Le mobilier métallique issu des fouilles anciennes n'a pas été intégralement conservé, ce qui conduit à une sous-évaluation, en particulier des objets indéterminés ou non identifiés qui n'apparaissent pas ou peu dans nos inventaires. Ceci explique pour une bonne part les différences observées dans les données quantitatives brutes ${ }^{6}$. Le mobilier décompté dans l'ESS 7 provient ainsi en partie de ces fouilles anciennes. Cette réserve est moins sensible dès lors que l'on aborde ce mobilier d'un point de vue qualitatif, car les objets indentifiables ont été, dans tous les cas conservés et présentés dans les mémoires qui ont rendu compte des découvertes (Marc et Vialard, 1973).

Le mobilier métallique issu des ESS 5 et 7 a été découvert aux deux tiers dans les bâtiments, quelle que soit leur fonction, contre un tiers seulement dans les diverses cours. Ce rapport s'inverse quasiment par rapport à la céramique, qui est massivement rejetée hors des bâtiments du fait de l'entretien régulier des sols. Le mobilier métallique serait donc conservé, perdu ou abandonné de manière privilégiée dans les bâtiments, ce qui suppose qu'il y ait été entreposé assez systématiquement, éventuellement même lorsqu'il était hors d'usage. Cela suggère que ces objets aient présenté un intérêt particulier pour les occupants et qu'on peut, dès lors, les mettre en relation étroite avec leur contexte de découverte. Leur degré de pertinence par rapport à la question que nous nous posons ici serait donc élevé, même si une pratique de la récupération de mobilier exogène n'est pas à exclure. La présence de ces objets peut être mise en rapport avec l'usage que l'on en faisait sinon dans la pièce ou le bâtiment où ils ont été mis au jour, du moins dans l'ESS à laquelle ils appartiennent.

La conservation de ce genre de matériel usé, cassé, s'explique : par la perte involontaire qui a permis leur découverte sur les sols intérieurs et extérieurs; par leur rejet alors qu'ils sont hors d'usage - ils sont découverts pour partie dans des dépotoirs, rejetés dans un coin des cours ou des bâtiments ; par leur stockage volontaire avant réparation et réutilisation, éventuellement, détournée de leur usage initial.

Les ESS 5 et 7 sont celles qui contiennent le plus grand nombre d'objets métalliques, respectivement 124 et 145 en nombre de restes déterminé (NRD) pour 164 et 176 en NR brut, soit un taux de détermination respectivement de $75,60 \%$ et $82,30 \%$. Ce mobilier est attribuable aux phases $2 \mathrm{C}$ à $2 \mathrm{E}$, soit entre le début du XIV ${ }^{\mathrm{e}}$ et jusqu'au XV ${ }^{\mathrm{e}}$ siècle.

Le cadre de classement que nous avons retenu reste toujours discutable, mais nous avons pris le parti de limiter le nombre de catégories à six (fig. 8). Les clous, qui ne sont pas toujours interprétables avec certitude, sont comptabilisés dans la catégorie " harnachement » lorsque des clous de ferrage sont identifiés, ou pour les autres dans la catégorie "Construction et autre quincaillerie ", qui constitue un groupe donc très largement ouvert. Nous avons défini les catégories suivantes : 1/ Outils et ustensiles; 2/Équipement domestique; 3/Construction et autres éléments de quincaillerie; 4/ Vêtement et parure ; 5/ Harnachement/chasse ; 6/ Fragments indéterminés. 
Figure 8 : Répartition comparée du mobilier métallique des ESS 5 et 7, par catégorie Figure 8: Compared distribution of the metallic objects of the ESS 5 and 7, by category.

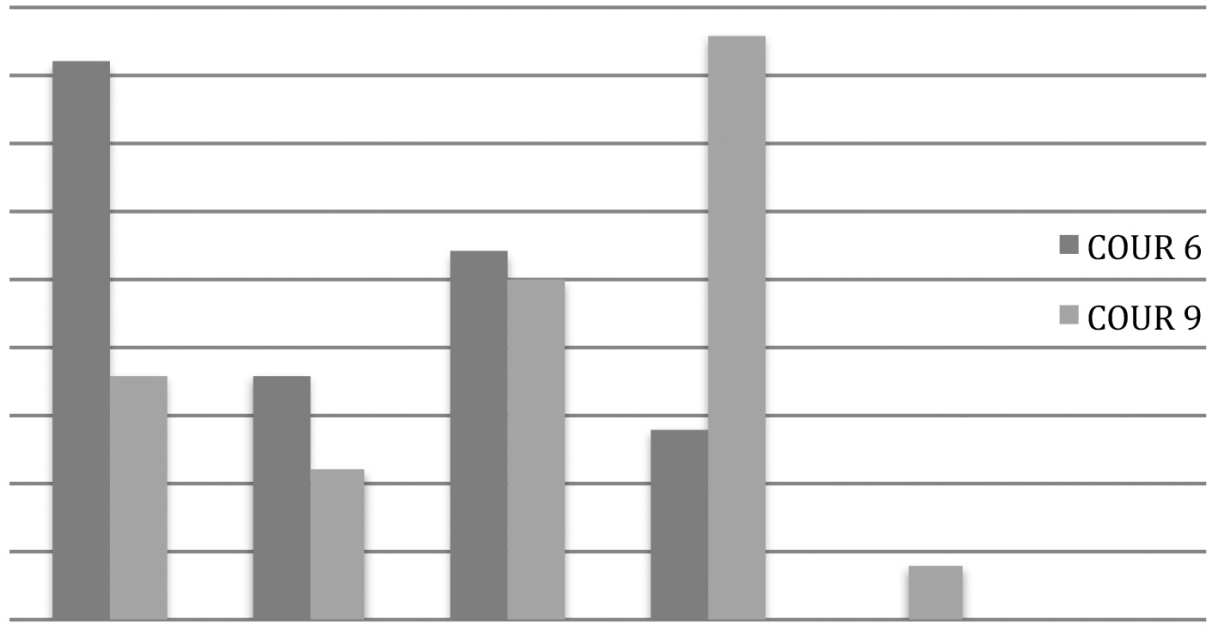

Si le fer compose la matière première de la majorité des objets (tant en NR, qu'en poids), cela va de soi. Le mobilier en alliage cuivreux comprend de petits objets assez communs sur les sites de cette époque : boucles, plaques décoratives, dés à coudre, fil, mordant de ceinture, serrurerie de coffre, etc.; il est présent dans des proportions équivalentes dans les deux ESS.

Sur l'ensemble du site, une soixantaine d'objets différents sont individualisés, mais seulement quarante-trois sont dénombrés dans les ESS 5 et $7^{8}: 20$ dans l'ESS 5, 35 dans l'ESS 7 (fig. 8). Plus que les données quantitatives, celles qui nous intéressent ici sont les données qualitatives, qui autorisent quelques pistes d'interprétation sur les différentes structures bâties ou sur les activités potentiellement pratiquées dans ces ESS. Toutes les rubriques de notre cadre de classement contiennent au moins une occurrence, mais au regard de la question que nous traitons ici, toutes ces catégories n'ont pas le même intérêt (fig. 9). La présence ou l'absence de certains objets dans les catégories qui indiquent la pratique d'activités spécialisées - en tant qu'elles ne relèvent pas du bricolage ou de travaux quotidiens - nous intéresse plus que d'autres. Nous ne nous attarderons pas sur la présence d'animaux ferrés, attestée de manière directe avec des fers à cheval (7 dans l'ESS 7), ou indirecte avec des clous de fer à cheval (9 dans l'ESS 5). Ceci a contribué à l'interprétation hypothétique du bâtiment 11 (ESS 5) et de la pièce 2 du bâtiment 12 (ESS 7) comme une écurie. C'est la catégorie «Outils et ustensiles » qui retient d'abord notre attention. Nous sommes parfaitement conscientes qu'il s'agit d'indicateurs fragiles car numériquement faibles, mais il s'agit de la réalité documentaire inhérente à l'échelle à laquelle nous nous sommes placées. 
Figure 9 : Dénombrement des objets métalliques dans les ESS 5 et 7 Figure 9: Inventory of metallic objects in the ESS 5 and 7.
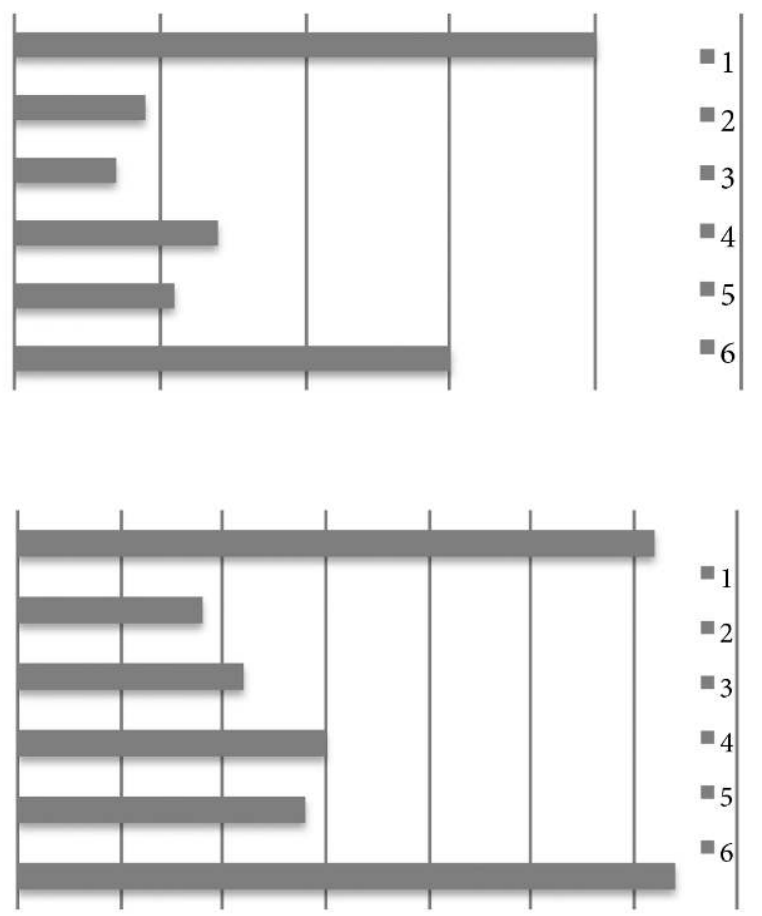

1. Outils et ustensiles ; 2 . Équipement domestique ; 3 . Construction et autre quincaillerie ; 4 . Vêtement et parure ; 5 . Harnachement /chasse ; 6 . Indéterminé

Premier constat (tab. 1, fig. 10) : il y a deux fois plus de sous-catégories d'outils dans l'ESS 7 que dans l'ESS 5 (10/5) En première instance, cette information suggère une plus grande diversité des activités pratiquées au sein de cette entité que ni la taille de ces deux ESS, ni leur durée de fonctionnement, ne peuvent à elles seules expliquer.

Tableau 1: Aperçu du mobilier métallique mis au jour dans les ESS 5 et 7 Table 1: Overview of metallic artefacts found in the ESS 5 and 7.

\begin{tabular}{|l|l|l|l|}
\hline \multicolumn{2}{|l|}{} & ESS 5 & ESS 7 \\
\hline \multicolumn{2}{|l|}{ Outils et ustensiles } & & 1 \\
\hline 1 & Burin & & 1 \\
\hline 2 & Ciseaux à bois & & 1 \\
\hline 3 & Couteau & 1 & 1 \\
\hline 4 & Émondoir & & 1 \\
\hline 5 & Fil de bronze & & 1 \\
\hline 6 & Fil de cuivre & 1 & \\
\hline 7 & Gouge & & \\
\hline
\end{tabular}




\begin{tabular}{|l|l|l|l|}
\hline 8 & Lame & & 3 \\
\hline 9 & Plomb (fragment) & 6 & \\
\hline 10 & Serpette & 2 & \\
\hline 11 & Soc & & 2 \\
\hline 12 & Tige & & \\
\hline 13 & Virole & 22 & 19 \\
\hline
\end{tabular}

Equipement domestique

\begin{tabular}{|l|l|l|l|}
\hline 14 & Applique & 9 & 5 \\
\hline 15 & Chandelier à broche & & 1 \\
\hline 16 & Clef & 2 & 5 \\
\hline 17 & Dé à coudre & & 2 \\
\hline 18 & Lampe à huile & & 1 \\
\hline
\end{tabular}

Construction et autres éléments de quincaillerie

\begin{tabular}{|c|c|c|c|}
\hline 20 & Anneau & 2 & 3 \\
\hline 22 & Chaînette & & 1 \\
\hline 23 & Clou & 53 & 64 \\
\hline 24 & Crochet & 1 & \\
\hline 25 & Disque & & 1 \\
\hline 27 & Ferrure & & 3 \\
\hline 28 & Fiche & & 1 \\
\hline 29 & Gond & 1 & 1 \\
\hline 31 & Penture & 2 & 2 \\
\hline 32 & Plaque fer & 6 & 3 \\
\hline 33 & Plaque plomb & 1 & \\
\hline 34 & Serrure & 1 & \\
\hline & & & \\
\hline
\end{tabular}


31 bois) démontre un bon niveau d'équipement de cette unité et illustre surtout la multiplicité des activités qui y sont pratiquées. Ces capacités s'enrichissent encore de celle du tissage, attestée dans le bâtiment 17 . Les objets les plus emblématiques sont deux socs de charrue très usés, dont un découvert dans la fosse de stockage du bâtiment 12 (pièce 4 interprétée comme une cuisine), un autre au cours des fouilles des années soixante-dix. On pourra nous objecter que le soc ne fait pas la charrue, ou du reste ne garantit pas l'emploi de la charrue par les détenteurs de cette ESS. Mais cet objet n'est ni plus ni moins pertinent que n'importe quel autre objet mis au jour sur le site...

L'ESS 5 est très différemment dotée. Bien qu'aucun bâtiment d'exploitation n'y ait été localisé, hors l'hypothétique écurie, son niveau d'équipement global en mobilier en fer est tout à fait correct. Cependant, parmi les outils, seuls un émondoir et une serpette ont pu être identifiés. Ces derniers suggèrent la pratique de la taille des arbres, fruitiers ou plessés, mais rien ne permet de préciser leur usage exact. Ce qui retient également l'attention est que l'occupant de cette maison conservait ce que nous pouvons qualifier de «réserve de plomb» qui se présente en particulier sous la forme d'une bande de $4,7 \mathrm{~cm}$ de large enroulée autour d'un clou, le tout pesant $306 \mathrm{~g}$, ainsi que d'une petite plaque repliée sur elle-même de $60 \mathrm{~g}$. Il est possible de qualifier ces matériaux de "plomb vieux", fruits d'une récupération, mais que faisait-on de cette matière première? La présence d'une gouttelette de plomb suggère une activité de transformation, qu'aucun autre indice ne vient renseigner davantage, interdisant d'orienter l'interprétation vers la pratique d'une activité de production artisanale, plutôt que vers celle d'un usage opportuniste, de « bricolage » en quelque sorte, à partir de cette matière première.

Quoi qu'il en soit de cette dernière information, bien délicate à manier au demeurant, il apparaît que le mobilier métallique mis au jour dans ces deux ESS relevant de la catégorie "Outils», renvoie, de par sa composition, une image bien différente des travaux pratiqués dans ces deux entités. Les éléments convergent pour faire de l'ESS 7 le centre d'une activité diversifiée, assimilable à celle d'une exploitation agricole: travail du sol, bricolage centré sur la menuiserie, production textile à partir de la laine de moutons élevés localement, ce qui ne saurait surprendre dans une région de céréaliculture dominante qu'est la plaine de Caen au bas Moyen Âge. Dans le second cas, la présence de plomb et d'autres menus fragments pourrait suggérer une activité que l'on n'a pas osé néanmoins qualifier d'artisanale faute d'indices suffisants. Nonobstant, cette incertitude, l'impression que l'activité agricole n'est pas dominante au sein de l'ESS 5, résulte du niveau d'équipement agricole. Cela pourrait renvoyer implicitement à l'idée que la maisonnée serait celle d'un modeste paysan. Mais le reste du mobilier métallique présent dans cette entité ne distingue guère celle-ci de l'autre, ni par son volume, ni par sa nature qui ne suggère aucune débilité évidente des ressources, bien que cette notion reste très délicate à manier. Certes, dans l'ESS 7, la présence d'une petite bague à chaton, découverte dans le bâtiment 17, et d'une petite applique vestimentaire, objet complexe doté d'un décor architecturé formant un dais gothique (non représenté) esquisse une légère différence qualitative entre les deux ensembles. Mais, la découverte, dans la pièce accueillant les foyers (pièce 1) de l'ESS 5, d'une pierre sculptée au décor anthropomorphe relevant d'un «style naïf ", n'ayant aucune ou une faible valeur marchande, à supposer que sa fabrication relève d'un 
achat. Surtout, la signification de cet objet nous échappe : sorte d'enseigne évoquant la fonction du propriétaire des lieux? Valeur décorative tel un témoignage de la personnalité de son propriétaire? (fig. 10). L'argument économique et/ou social ne peut donc être convoqué trop vite pour expliquer ces différences.

Figure 10 : ESS5, Bâtiment 9

Figure 10: ESS 5, building 9.

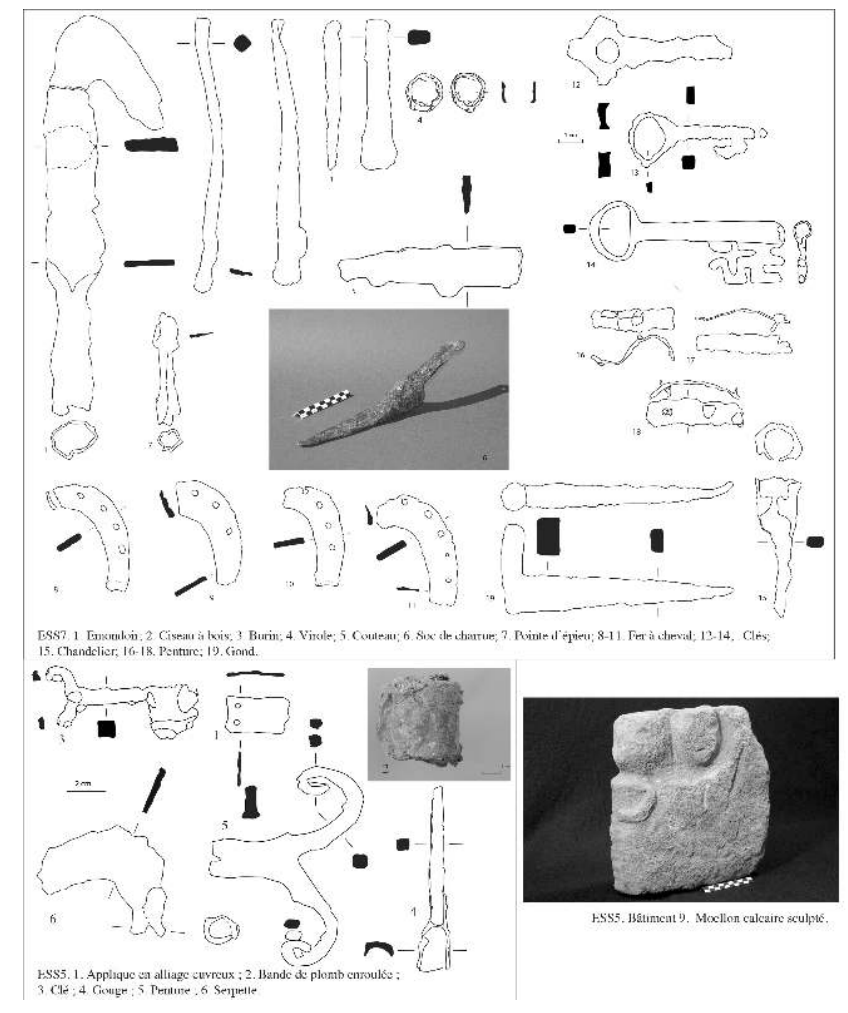

\section{Conclusion}

Au terme de cet exposé des faits, de ces constats limités et prudents, il convient de confronter ces différents indicateurs, de les mettre en perspective pour évaluer la réponse à notre interrogation initiale et la pertinence de notre démarche.

Les deux ESS que nous avons traitées, comparées, opposées au long de ces pages, présentent des différences structurelles claires : leur dimension, leur composition, la nature des bâtiments, les distinguent avec évidence. Soyons claires : ce premier niveau d'observation engage notre lecture des données. L'étude du mobilier céramique, outre le fait qu'elle permet de confirmer que ces deux ensembles ont bien fonctionné durant les mêmes phases, éventuellement de manière partiellement synchrone, ne révèle cependant aucune différence significative quant au niveau d'équipement de ces deux unités, dans la mesure bien sûr où l'on accepte que la gestion des déchets n'introduit aucun biais dans la représentativité de ces matériels. De ce point de vue, la céramique apparaît donc, dans notre contexte, comme un marqueur non distinctif, en somme faiblement pertinent par rapport à notre question. En revanche, le mobilier métallique introduit, malgré la faiblesse numérique des échantillons, une distinction sur les activités pratiquées, les pratiques agricoles, le travail de la charrue notamment, 
associées à une activité de travail du bois, une menuiserie domestique ? Ce sont autant d'éléments qui renvoient à la multitude des compétences requises dans le fonctionnement d'une unité d'exploitation agricole au quotidien. De l'autre côté, nous avons à faire à une unité dont les activités agricoles n'apparaissent pas clairement, ce qui suggère que l'ESS 5 n'est pas a priori le centre d'une unité d'exploitation agricole. Bien entendu, il faut se garder de généraliser ce bilan à d'autres sites.

Le niveau d'équipement respectif des deux ESS, tel que nous l'avons restitué, ne permet guère de fonder une quelconque hiérarchie économique, sur des arguments tangibles, qui expliquerait l'absence de certains outils, par exemple dans l'ESS 5. Nous nous garderons, en effet, de fonder une hiérarchie économique et a fortiori sociale, sur le seul constat des différences que les pages qui précèdent ont mis en évidence. Nous ne corrélons plus aujourd'hui la taille d'une unité au statut de son occupant, selon la hiérarchie que révèlent les textes : vavassorie, villainage, bordage, comme cela fut le cas antérieurement sur le modèle des premières interprétations proposées, par exemple, sur le site de Wharram Percy et comme nos collègues britanniques continuent à le pratiquer. Certes, nous pouvons formuler l'hypothèse que les mètres carrés consacrés au stockage des récoltes sont relatifs aux superficies travaillées, et donc que le tenant de l'ESS 7 dispose d'une exploitation plus grande que celle sur laquelle travaillent d'autres villageois qui occupent, dans le village de Courtisigny, des ensembles plus modestes en apparence (ESS 8, ESS 6, etc.). Nous sommes donc conduits à interpréter toutes les différences, que nous avons répertoriées comme des marqueurs de distinction fonctionnelle de ces unités - ce que la lecture du bâti suggérait d'ailleurs initialement. Cette lecture strictement fonctionnaliste de notre documentation peut être jugée pauvre, mais elle s'attache aux seuls faits et tend à échapper à une lecture trop étroitement historiciste. Le chat est maigre, mais à l'échelle à laquelle nous situons nos analyses, celle de l'unité de vie, qui met hors de portée le reste des possessions, notamment les terres exploitées, les jardins vivriers, en bref tout ce qui contribue à assurer la consommation quotidienne, à construire l'équilibre économique au long de l'année, échappe à nos investigations. L'analyse des restes fauniques même peu abondants devrait être systématiquement pratiquée sur ces sites récents. Notre lecture est certes pointilliste, fondée sur des observations de détail, sur de petits nombres, mais c'est clairement en croisant ces marqueurs, aussi ténus soient-ils, que nous pouvons espérer contourner les limites de notre information afin d'atteindre les modestes habitants de nos villages.

\section{BIBLIOGRAPHIE}

BOCQUET-LIÉNARD A., DUFOURNIER D., LOUIS-PHILIPPE M., et DERVIN S., 2011 - « Céramiques des XIVe $\mathrm{XV}^{\mathrm{e}}$ siècles provenant de dépotoirs (puits et latrines) du square Montoir-Poissonnerie (Caen, Calvados) », in BOCQUET-LIÉNARD A. et FAJAL B., À propos de l'usage de la production et de la circulation des terres cuites dans l'Europe du nord-ouest (XIV ${ }^{e}-X V I^{e}$ siècle), Actes de la table ronde du CRAHAM n ${ }^{\circ} 5$, Publications du CRAHM, Caen, p. 245-276. 
BOUGARD F. et NOYÉ G., 2003 - « Archéologie médiévale et structures sociales. Encore un effort », in BARTHÉLÉMY D. et MARTIN J.-M. (éd.), Liber largitorius : études d'histoire médiévale offertes à Pierre Toubert par ses élèves, Genève, Droz, coll. « Hautes études médiévales et modernes », 84, p. 331-346. DAVIES W., 1988 - Small worlds: the village community in early medieval Brittany, University of California Press.

DEMIERRE et al., 2013 - « La quantification des mobiliers d'instrumentum », B. FORT et N. TISSERAND (dir.) Le mobilier métallique et l'instrumentum : approches méthodologiques ", Les Nouvelles de l'archéologie, $\mathrm{n}^{\circ}$ 131, Dossier spécial, p. 10-14.

DERVIN S, HANUSSE C. et BOCQUET-LIÉNARD A., 2013 - « Approvisionnement en céramiques d'un habitat rural de la plaine de Caen au Moyen Âge. Approche pluridisciplinaire », Des productions céramiques de l'époque gallo-romaine à la Renaissance, Actes des Journées d'étude de Fosse et Amiens (2008-2009-2010), Revue archéologique de Picardie, $\mathrm{n}^{\text {os }}$ 1-2, p. 165-178.

DERVIN S., 2015 - « La céramique », in GUILLOT B. (dir.), Forges médiévales et écurie de la renaissance au château de Caen, Caen, [Publications du CRAHAM], Presses universitaires de Caen, p. 219-250.

FLAMBARD HÉRICHER A.-M. (dir.), BOCQUET-LIÉNARD A. et DERVIN S., avec la collaboration de HINCKER V., 2008 - Projet collectif de recherche : Typo-chronologie de la céramique médiévale dans l'espace bas-normand du $\mathrm{X}^{\mathrm{e}}-\mathrm{XVI}^{\mathrm{e}}$ siècle. Production, diffusion, Rapport 2008, Caen, non publié, $167 \mathrm{p}$.

FLAMBARD HÉRICHER A.-M. (dir.), BOCQUET-LIÉNARD A. et DERVIN S. avec la collaboration de HINCKER V., 2009 - Projet collectif de recherche : Typo chronologie de la céramique médiévale dans l'espace basnormand du Xe ${ }^{\mathrm{e}}-\mathrm{XVI}^{\mathrm{e}}$ siècle. Production, diffusion, Rapport 2009, Caen, non publié, 180 p.

HANUSSE C., 2012 - Le village de Saint-Ursin de Courtisigny : le site des fosses Saint-Ursin, Habilitation à diriger les recherches, Université de Paris I, Panthéon-Sorbonne, volume 2-1-novembre, 243 p., volume 2-2, 73 pl., non publié.

HANUSSE C. et T. JARRY, 2007 - «Espace bâti et habitation en Normandie (Plaine de Caen) du XIII ${ }^{\mathrm{e}}$ au $\mathrm{XV}^{\mathrm{e}}$ siècle. Mise en regard des sources archéologiques et textuelles ", in MADELINE Ph. et MORICEAU J.-M., (éd.), Bâtir dans les campagnes. Les enjeux de la construction, de la protohistoire au XIXésiècle, Caen pôle rural-MRSH, Presses universitaires de Caen, p. 133-152.

MARC J.-C. et VIALARD P., 1973 - Fouille sur le site du village médiéval de Saint-Ursin de Courtisigny (chantier Ouest 1), Université de Caen, Caen, non publié, 2 vol.

OLIVIER L., 2008 - Le sombre abîme du temps : mémoire et archéologie, Paris, Seuil.

SMITH S., 2009 - « Towards a social archaeology of the late medieval English peasantry: Power and resistance at Wharram Percy », Journal of Social Archaeology, 2009-9, p. 391-416.

\section{NOTES}

1. «Il est important, cependant, que les archéologues n'abandonnent pas l'étude de ces questions [les thématiques économiques et sociales] aux historiens ; nous devons reconnaître que les deux types de sources écrites et matérielles ont joué un rôle dans la production de données et la restitution des réalités de la vie paysanne, et que les deux catégories avaient à voir avec les relations de pouvoir et la création de la vie sociale. Ces deux types de données doivent être traitées côte à côte, aucune n'ayant de pouvoir d'explication supérieur à l'autre. » 
2. Ces aménagements massifs justifient la dénomination de «fosses » accolée à celle de saint Ursin auquel était certainement dédiée l'église paroissiale. Ce toponyme qui se fixe à l'époque moderne était l'ultime indice de la présence d'un village disparu.

3. Au pied des versants de la fosse, les niveaux archéologiques sont plus puissants du fait de l'accumulation de couches d'équilibre à chaque aménagement ou reprise de versants; leur stabilisation est assurée, dans quelques cas documentés, grâce à la construction d'un mur de soutènement qui peut constituer le cas échéant le mur de fond d'une cour.

4. Sur tous ces aspects nous pouvons renvoyer commodément à la réflexion théorique de L. Olivier (Olivier, 2011).

5. Ces formes en grès du Mortainais/Domfrontais se retrouvent régulièrement sur les sites caennais dès la fin du XIII ${ }^{\mathrm{e}}$ siècle et notamment au château de Caen (Dervin, à paraître).

6. Il semble qu'un tri ait été effectué sur le terrain ou immédiatement après.

7. Le taux supérieur de l'ESS 7 s'explique pour les raisons évoquées dans la note précédente.

8. Les figures représentent la totalité des objets identifiés, ce qui permet de mieux situer ce qui est présent ou absent dans les ESS étudiées ici.

\section{RÉSUMÉS}

L'article explore nos capacités d'interprétation fonctionnelles de deux entités spatiales structurées, des unités parcellaires bâties, appartenant au site des "Fosses Saint-Ursin » à Courseulles-sur-Mer (Calvados), datables des $\mathrm{XIV}^{\mathrm{e}}$ et $\mathrm{XV}^{\mathrm{e}}$ siècles. L'analyse se fonde sur une confrontation de plusieurs indicateurs : le nombre et la composition des unités d'habitation et des unités d'exploitation, le mobilier céramique et le mobilier métallique découverts. Seul le croisement de différents marqueurs autorise l'interprétation de ces ensembles, qui reste à ce stade limité à sa dimension fonctionnaliste, et se tient volontairement à distance d'une approche étroitement historiciste.

This paper explores our capacity to interpret two structured spatial entities, built units, from the 14th-15th century, from the site of "Fosses Saint-Ursin" in Courseulles-sur-Mer (Calvados). The analysis is based on the confrontation of several indicators: the number and the composition of the inhabited units and exploitation units, the pottery and metallic artefacts discovered within. The use of these indicators enables the interpretation of these entities, which stays at this point limited to its functionalist dimension and voluntarily distances itself from historicisme.

Dieser Beitrag untersucht unsere Möglichkeiten einer Funktionsbestimmung von zwei strukturierten Einheiten, bebauten Flurstücken, die der Siedlung der „Fosses Saint-Ursin“ bei Courseuelles-sur-Mer (Calvados, France). Die Analyse stützt sich auf die Gegenüberstellung verschiedener Indikatoren: die Anzahl und die Zusammensetzung der jeweiligen Wirtschaftseinheiten, das Keramikinventar und die Metallfunde. Ausschließlich der Vergleich 
der verschiedenen Indikatoren ermöglicht eine wissenschaftlich zufriedenstellende Interpretation der Fundensembles, die jedoch in diesem Stadium auf ihre funktionelle Dimension begrenzt bleibt und die sich damit bewusst auf Distanz zu einer engen historisierenden Herangehen hält.

El artículo explora nuestras capacidades de interpretación funcionales de dos entidades espaciales estructuradas, las unidades fragmentadas construidas, pertenecientes al sitio "Fosses Saint-Ursin" en Courseulles-sur-Mer (Calvados), fechado en los siglos XIV y XIV. El análisis se basa en una comparación de varios indicadores: el número y la composición de unidades de vivienda y unidades operativas, los artefactos de cerámica y el material metálico descubiertos. Sólo el cruce de diferentes marcadores permite la interpretación de estos conjuntos, que sigue siendo limitada en esta etapa a su dimensión funcionalista, y se mantiene deliberadamente alejado de un enfoque estrechamente historicista.

INDEX

Mots-clés : Normandie, analyse spatiale, Moyen Âge

Schlüsselwörter : Normandie, mittelalterlich, räumliche Analyse

Palabras claves : Normandía, medieval, análisis espacial

Keywords : Normandy, medieval, spatial analysis

\section{AUTEURS}

CLAIRE HANUSSE

CRAHAM, UMR 6273, UCBN-CNRS

STÉPHANIE DERVIN

INRAP, CRAHAM, UMR 6273, UCBN-CNRS 